\title{
Des cheminotes en usine : des femmes aux ateliers d'Arles (1914-1920)
}

Railwaywomen in factory: women in the workshops of Arles (1914-1920)

David Lamoureux

\section{OpenEdition}

\section{Journals}

Édition électronique

URL : https://journals.openedition.org/rhcf/1794

DOI : $10.4000 /$ rhcf. 1794

\section{Éditeur}

Rails \& histoire

\section{Édition imprimée}

Date de publication : 1 décembre 2003

Pagination : 403-419

ISBN : 0996-9403

ISSN : 0996-9403

\section{Référence électronique}

David Lamoureux, "Des cheminotes en usine : des femmes aux ateliers d'Arles (1914-1920) », Revue d'histoire des chemins de fer [En ligne], 28-29 | 2003, mis en ligne le 31 décembre 2014, consulté le 22 avril 2022. URL : http://journals.openedition.org/rhcf/1794 ; DOI : https://doi.org/10.4000/rhcf.1794 


\section{Des cheminotes en usine : des femmes aux ateliers d'Arles (1914-1920)}

\section{Les femmes aux chemins de fer avant 1914}

Dès $1847^{1}$, des femmes travaillent dans les chemins de fer, mais leur entrée ne s'y fait pas sans réticence. Tout au long du XIX ${ }^{e}$ siècle et au début $\mathrm{du} \mathrm{xx}^{\mathrm{e}}$ siècle, leur emploi par les compagnies reste marginal. Elles sont cantonnées à certaines professions : gardes-barrières, employées de bureau ou, parfois, chefs de halte ou de station.

En 1885, pour la Compagnie des chemins de fer de l'Est, « L'accession des femmes aux carrières et emplois précédemment réservés à l'homme seul, doit être, en effet, restreinte, à [son] avis dans des bornes assez étroites, qui ne peuvent être fixées raisonnablement que par les lois de la nature et le sentiment des convenances $»^{2}$, et de préciser que : «dans la famille en possession de son chef, c'est-à-dire en toutes circonstances normales et régulières, l'homme doit suffire par son travail à faire vivre les siens, tandis que la femme, toujours attachée au foyer, doit borner son activité aux soins du ménage et des enfants ${ }^{3}$.»

Un point de vue partagé au début du siècle par les agents des chemins de fer réunis en syndicat, pour lesquels s'impose « l'interdiction absolue d'employer des femmes en dehors des gardes-barrières et des préposées à la salubrité [mais de] réserver des emplois féminins aux veuves ou filles d'agents et d'en exclure les femmes dont le mari est employé $»^{4}$. Ainsi, aussi bien pour la direction que pour les employés des chemins de fer, l'emploi de femmes doit rester exceptionnel et être un recours seulement en cas d'absolue nécessité tant pour la candidate que pour les compagnies. Cette situation connait un statu quo jusqu'au conflit de 1914.

1- Georges Ribeill, «1847 : les femmes entrent en gare... », La Vie du Rail, n 2196 (25 mai 1989), p. $45-48$.

2- Marcel Lemercier, «De l'emploi des femmes dans les chemins de fer français et spécialement à la Compagnie de l'Est», Revue générale des chemins de fer, janvier 1885, p. 20.

3- Ibid., p. 21.

4- Madeleine Guilbert, Les Femmes et l'organisation syndicale avant 1914, Paris, CNRS, Travaux du centre d'études sociologiques, 1966, p. 89. D'après le compte rendu du douzième Congrès national des cheminots de 1901. 


\section{L'arrivée des femmes avec la guerre}

Le déclenchement de la guerre, en 1914, bouleverse l'organisation des chemins de fer. En plus de fournir des efforts sur le trafic ferroviaire - les trains doivent transporter les hommes mobilisés de la France entière vers le Front - les réseaux doivent aussi s'intégrer dans un processus de production de matériel militaire. Les ateliers du service du Matériel de la Compagnie PLM sont transformés pour pouvoir exécuter de nouvelles tâches.

L'Administration de la Guerre a réclamé pour des fabrications intéressant la défense nationale une aide que nous avons donnée. Nous avons ainsi organisé dans nos ateliers de machines la fabrication des projectiles ; nous avons mis un certain nombre de nos ouvriers à la disposition de l'Arsenal de Lyon et nous avons accepté de recuire des obus pour cet établissement; nous avons exécuté des roues et des jantes pour les équipages militaires, des voitures à munitions, des wagonnets pour les transports des obus etc. $^{5}$

Tous les lieux de fabrication et de réparation du service du Matériel de la compagnie PLM sont mis à contribution : «dans les ateliers de Paris, d'Oullins et d'Arles, s'improvise, à la demande de la Guerre qui manque de munitions, la fabrication d'obus, de bombes, de pelles, de pioches etc. ${ }^{6}$. » Mais très rapidement le manque de maind'œuvre - beaucoup d'agents sont partis à la guerre - pousse la compagnie à embaucher du personnel féminin pour effectuer des tâches jusque-là dévolues aux hommes. Des femmes entrent aux ateliers d'Arles et deviennent des cheminotes en usine.

Le nombre d'emplois féminins dans les ateliers d'Arles est difficile à connaitre faute du registre du personnel pour les années 1914-1919 qui n'a pu être retrouvé. Grâce à celui du syndicat des chemins de fer CGT d'Arles, nous savons qu'au moins 126 femmes y ont travaillé entre janvier et juillet $1918^{7}$. Il s'agit d'un chiffre minimum car rien ne permet d'affirmer que toutes les femmes employées aux ateliers aient adhéré au syndicat. En février 1920, elles ne sont plus que 48 portées

5- Conseil d'administration du PLM (rapports annuels du conseil d'administration du PLM présentés à l'assemblée générale des actionnaires. Documents consultables pour le Sud-Est de la France aux archives de la CCI de Marseille et à la bibliothèque universitaire de droit de Montpellier, désormais : C.A. P.L.M.), 1915, p. 46.

6- Anonyme, «Le P.L.M. de la Guerre de 1914 à nos jours », Bulletin P.L.M., $\mathrm{n}^{\circ} 38$ (mars 1935), p. 53.

7- Le syndicat d'Arles possède les registres nominatifs de ses adhérents d'août 1917 à janvier 1920. Aucune femme n'adhère au syndicat avant janvier 1918. 
sur la liste du personnel tenue par la compagnie ${ }^{8}$. Parmi ces travailleuses, nous n'avons retrouvé des dossiers d'affiliation à la caisse de retraite que pour 39 d'entre elles, aux archives du personnel de la SNCF de Béziers ${ }^{9}$, soit 79,6\% des femmes recensées par la compagnie au début de février 1920 et, à titre d'indication, 30,9\% des cheminotes syndiquées en 1918. L'absence de certains dossiers s'explique par le fait qu'une partie de ce personnel féminin est encore stagiaire en $1920^{10}$. C'est avec cet échantillon, certes incomplet, composé de 39 femmes embauchées aux ateliers d'Arles entre 1915 et 1918 que nous allons essayer de mieux connaitre ce personnel marginal des ateliers d'Arles.

\section{L'entrée à la compagnie}

L'entrée des femmes aux ateliers s'est faite de façon échelonnée dans le temps, et après une période de flottement car aucune ne rejoint la Compagnie PLM en 1914. Si elles ne sont que quatre en 1915, trois en 1916 et 1917, en revanche, 29 cheminotes sont embauchées en 1918. Pour cette dernière année, une note du service du Matériel de 1918 précise, pour cinq travailleuses, qu'il s'agit d'une date d' "entrée fictive » à la compagnie. En fait, nous pensons qu'il pourrait s'agir d'une campagne de régularisation de personnes embauchées antérieurement par l'entreprise, ce qui relativiserait l'importance des embauches de 1918, et les répartirait sur une période plus longue.

Indéniablement, dès 1915, la compagnie fait appel à la maind'œuvre féminine, «tandis que le trafic reprenait l'allure des périodes les plus actives et les plus difficiles, l'effectif de notre personnel se trouvait amoindri. Pour faire face à ce trafic, nous avons dû recourir à une extension du personnel féminin ; nous avons aussi obtenu et utilisé sur certains points des prisonniers de guerre, et même nous avons fait appel à la main-d'œuvre militaire en cas d'impérieuse nécessité »" ${ }^{11}$. Malgré ces premières embauches, la situation continue à se détériorer : « les réseaux

8- Liste du personnel retrouvée aux ateliers d'Arles. Cette liste, établie par la direction du PLM en février 1920 et révisée durant la grève du mois de mai, est déposée au CE Cheminots PACA à Marseille.

9- Dossiers de la Caisse de Prévoyance et de Retraite de la SNCF concernant le personnel de la Compagnie du PLM, enregistrés sous la cote 1999/013/PLM. Ce fonds est constitué de dossiers d'agents partis sans pension de retraite et donc sortis de façon extraordinaire. La fiche d'affiliation du cheminot comporte des éléments utiles à l'historien : la date et lieu de naissance, l'emploi, la résidence, le traitement, et l'état civil. 10- L'affiliation des agents à la caisse de retraite n'intervenait que lorsqu'ils avaient effectué une période de stage d'un an.

11- C.A. P.L.M., 1916, p. 37. 
souffrent chaque jour davantage d'une pénurie de personnel ${ }^{12}$. » Rien d'étonnant à voir les femmes investir les différentes sections des ateliers d'Arles, car ces ouvrières font partie d'un plan de recrutement national qui doit pallier le manque de main-d'œuvre et permettre d'assurer la production. Mais si la compagnie recourt, chaque jour davantage, à la main-d'œuvre féminine, nous ne saurions dire dans quelle proportion.

Si l'entrée de ces femmes n'est pas anodine, aujourd'hui, il nous est difficile de retrouver les motivations qui les ont poussées vers les portes des ateliers. Néanmoins quelques éléments peuvent apporter un début de réponse. Le premier est la situation familiale des cheminotes. Lors de leur arrivée à la compagnie, les travailleuses célibataires sont les plus nombreuses : 15 , soit $38,5 \%$ des cas. Suivent les 14 femmes mariées $(35,9 \%)$ : neuf d'entre elles sont veuves $(23,1 \%)$, tandis qu'une seule est divorcée. Plus que ces situations détaillées, c’est le clivage opposant les femmes vivant seules à celles mariées qui est intéressant. En effet, la grande majorité des cheminotes embauchées aux ateliers n'ont pas ou plus de conjoint (64,1\% des cas), plus du tiers étant veuves. Quatre sont clairement identifiées comme des veuves de guerre, mais il y a de fortes probabilités pour qu'elles le soient toutes. En revanche, aucun élément ne laisse présager qu'elles aient été mariées à des cheminots $^{13}$. L'obtention d'un emploi dans les ateliers ne relèverait donc pas de la bienveillance de la Compagnie du PLM, contrairement à d'autres postes obtenus par des femmes d'agents décédés, dans les bureaux ou aux barrières. Hormis un cas isolé, les célibataires, non plus, n’ont pas de lien avec le monde du chemin de fer. Plus probablement, ces cheminotes, qu'elles soient veuves ou célibataires, doivent trouver un emploi pour se nourrir car elles ne peuvent espérer de revenus de quiconque. Une situation ressentie par un syndicaliste qui résume : «par le fait même de la guerre des veuves, des filles à marier, tout cela aura besoin de travailler, et cela à tout prix parce qu'il faudra, comme dit l'autre, que l'on mange à sa faim. Elles n'auront pas de mari qui apportera son salaire à la maison pour qu'elles puissent faire leur travail de ménagères : et alors elles iront sur le marché du travail. ${ }^{14} \gg$ Un discours écrit au futur en mai 1918 mais qui s'intègre bien dans le quotidien de

12- C.A. P.L.M., 1917, p. 40.

13- L'absence de lien entre le nom des femmes et celui des agents décédés, pendant la guerre, et inscrits sur la plaque commémorative des ateliers d'Arles, confirmerait cette hypothèse.

14- Union des syndicats du réseau PLM, Compte rendu sténographique du II congrès de l'Union des syndicats du réseau P.L.M, organisé par le Syndicat de Clermont-Ferrand, les 18, 19, et 20 mai 1918, 1919, Dijon, Imprimerie coopérative ouvrière, p. 120. 
ces années de guerre et qui illustre la précarité créée dans les foyers par le conflit et la mort des hommes au combat. Le besoin d'un salaire pour (sur)vivre semble bien être une des raisons qui amène les femmes aux ateliers. Mais au-delà de cette nécessité, la proximité des ateliers parait être un autre facteur déterminant dans cette volonté pour venir travailler à la Compagnie PLM.

\section{Origines géographiques et sociales des cheminotes}

En effet, la plupart des femmes embauchées sont méditerranéennes. 23 sont originaires des Bouches-du-Rhône (59 \% des cas), et $\mathrm{du}$ « cru » puisque 22 sont arlésiennes. Cinq ont vu le jour dans le département limitrophe du Gard (12,8 \% des cas), dans des communes proches d'Arles : Fourques, Saint-Gilles et Vallabrègues. Au total ce sont près de sept cheminotes sur dix qui sont nées à proximité des ateliers. Les autres employées ont des origines plus diverses. Hormis les deux Ardéchoises, chaque origine ne compte qu'une seule représentante, mais toutes sont situées sur les lignes du réseau PLM, en dehors des cheminotes nées en Corse et dans le Loir-et-Cher. En 1920, elles habitent toutes à Arles, sauf deux qui résident à Fourques. La proximité est donc bien un élément déterminant dans cette entrée aux ateliers qui sont le plus gros employeur de la ville. En revanche, le milieu social semble avoir moins d'importance.

Ces cheminotes sont issues, pour le grand nombre, d'un milieu rural. Quinze d'entre elles ont un père agriculteur à leur naissance et une, un jardinier (38,5\% des cas au total). Neuf pères sont artisans, commerçants ou ouvriers $(23,1 \%)$. Une grande diversité de professions apparait : hormis deux menuisiers, tous les métiers ne comptent qu'un seul représentant. Sept pères travaillent dans les « services » $(17,9 \%)$, trois seulement sont cheminots $(7,7 \%$ des cas), deux « journaliers », un «commis », et l'autre «cocher». Finalement, peu de femmes ont des origines dans les chemins de fer, moins de $8 \%$ d'entre elles ont un père cheminot. Deux sont nées de père inconnu, et pour six d'entre elles, nous n'avons retrouvé aucun élément éclairant la profession de leur géniteur. Si nous ne tenons compte des cas où nous ne connaissons pas l'activité du père, alors la répartition par secteur s'établit de la manière suivante. Près de la moitié des travailleuses ( $48 \%$ exactement) ont des origines rurales. La proximité d'une population rurale aux alentours d'Arles est une explication à ce phénomène. Suivent celles qui ont des pères artisans ou ouvriers $(29 \%)$ et celles dont le géniteur travaille dans les services $(22,6 \%)$. Ainsi, le monde des ateliers, proche de celui de l'usine, attire d'abord une main-d'œuvre aux origines rurales et ouvrières. Enfin l'âge est le dernier facteur auquel nous nous intéresserons. 


\section{L'âge à l'entrée à la Compagnie}

Aux ateliers, on ne trouve pas de très jeunes travailleuses, ni de vieilles ouvrières. Lors de leur embauche, si la moins âgée a 20 ans, à l'autre extrémité, la plus vieille a 39 ans et la moyenne d'âge est relativement élevée : 27,8 ans. Si nous opérons une répartition par tranches d'âges, 11 cheminotes entrantes ont entre 20 et 24 ans, 13 entre 25 et 29 ans, 12 entre 30 et 34, et trois plus de 35 ans. Les travailleuses célibataires ont la moyenne d'âge la plus faible, qui est tout de même de 25 ans, alors que celle des veuves est de 28 ans et celles des femmes mariées de 29 ans. L'absence de personnes de plus de 40 ans pourrait provenir du fait qu'a priori aucune femme ne travaillait aux ateliers avant la guerre et que la compagnie n'a pas embauché de travailleuses trop âgées. En revanche, le PLM n'applique plus, en cette période de guerre, l'âge limite d'admission aux chemins de fer qui était de 30 ans avant le conflit. Face à un manque chronique de main-d'œuvre, il ne se permet plus d'être aussi exigeant qu'avant la guerre.

\section{Travailler aux ateliers $d^{\prime}$ Arles}

\section{Les emplois à la compagnie}

Cette entrée des femmes à la compagnie est un véritable bouleversement aux ateliers. Elles investissent un monde historiquement masculin. Elles sont là pour remplacer temporairement les hommes et exercent donc des métiers d'hommes. Aux ateliers, point d'employée de bureau ${ }^{15}$, ni de $"$ métiers de femmes $»^{16}$, mais des « conductrices de machines-outils », des « manœuvres », des « aides-soudeuses »... Les services administratifs de la compagnie hésitent sur la terminologie des métiers à utiliser. En effet, certaines fiches comportent une féminisation de la profession alors que d'autres ont gardé l'appellation masculine. Ainsi, toutes les femmes employées à la section de l'ajustage restent des " aides-ajusteurs », même situation pour l'« aide-chaudronnier », peutêtre un signe d'une misogynie plus forte dans ces sections que dans les autres. Il se produit, parfois, une hésitation ou peut-être un maintien d'habitudes ; ainsi perdure une masculinité pour une « aide-ferblantier » et deux « aides-tourneurs », alors que leurs autres collègues sont toutes « aides-ferblantières » ou « aides-tourneuses ». On a aussi une double

15- D'après le registre du syndicat en juillet 1918, neuf femmes travailleraient dans les bureaux, mais nous ne les retrouvons pas dans notre échantillon. Peut-être n'ont-elles pas interrompu leur carrière avant leur retraite.

16- En référence à l'article de Michelle Perrot, «Qu’est-ce qu’un métier de femmes ?», Le Mouvement social, $\mathrm{n}^{\circ} 140$ (juillet-septembre 1987), p. 3-9. 
appréciation pour la féminisation du terme "pilonneur» qui devient «pilonneuse » ou « pilonnière ». Globalement, malgré ces doubles emplois ou ces hésitations, c'est quand même la féminisation des termes employés qui prime dans 30 fiches d'affiliation sur 39 .

Les cheminotes les plus nombreuses sont « conductrices de machines-outils ", 10 sur 39 (25,6 \% des cas). D’après Maurice Bouvier-Ajam, "Afin de produire beaucoup plus, sans user trop de combustible, les industriels surent créer, renouveler ou perfectionner l'outillage. La généralisation des machines-outils fut importante dans la métallurgie, et dans d'autres fabrications $»^{17}$. Le travail simplifié par la machine permet de compenser le manque de qualification des femmes. Elles ne sont là que pour effectuer différentes opérations, leur travail ne requérant que peu de savoir-faire, à l'opposé de l'ajusteur, par exemple, qui doit utiliser différents outils. Parmi les autres femmes qui pourraient être aussi qualifiées d'ouvrières se trouvent cinq "pilonneuses», trois "lessiveuses-décapeuses", une " graisseuse de transmission », et une « tourneuse ». Sauf, pour cette dernière peut-être, les tâches confiées à ces cheminotes ne sont pas intéressantes : pilonner, laver ou graisser.

Le personnel féminin restant est « aide ». cinq occupent le poste $d^{\prime}$ « aide-ajusteur» et $d^{\prime}$ ' aide-ferblantière ». Trois exercent le métier d'« aide-soudeuse » ou d'« aide-tourneuse », et une celui d'« aide-chaudronnier ». Point commun entre toutes ces professions : un emploi subalterne qui ne requiert pas de qualification. Les femmes ont-elles passé un essai professionnel ? Rien n'est moins sûr. Elles sont plutôt des suppléantes des ouvriers de métier qui donnent « le coup de main », ou finissent les tâches, une fois les difficultés éliminées. Moins qualifiées que les aides, les femmes « manœuvres » ne sont que deux : pas de dénomination précise pour un travail imprécis, des cheminotes qui sont là pour tout faire.

Globalement, ces femmes apparaissent plutôt comme une maind'œuvre de «seconde main» dans la production. Qu'elles soient ouvrières, aides ou manœuvres, à aucune n'est demandé un travail qualifié, requérant savoir-faire et métier. L'utilisation de machines permet de compenser le manque de formation des femmes, car la compagnie ne veut pas en faire des ouvrières de métier. En 1918, un cheminot raconte qu'au Matériel « il y a des ateliers où il y a même des femmes peintres. Elles n'ont pas fait d'apprentissage, mais néanmoins elles savent passer

17- Maurice Bouvier-Ajam, Histoire du travail en France depuis la révolution, Paris, Librairie générale du droit de la jurisprudence, 1969, p. 254. 
le badigeon et cela suffit à la Compagnie $»^{18}$. La compagnie ne remplace par des femmes que la main-d'œuvre peu ou pas qualifiée. Elle pense peut-être comme le directeur de la Compagnie des Dombes trente ans plus tôt que : «La femme arrive à faire à la perfection, et sans s'en lasser, ce qu'elle fait tous les jours ; mais ne lui demandez pas de l'initiative ; n'oubliez pas que son rôle dans la vie n'est pas d'en avoir ${ }^{19}$. »

Les cheminotes s'insèrent, différemment suivant les sections, dans le processus de production des ateliers. La section des Machines-outils est celle qui compte le plus de femmes ( $7 \%$ des effectifs de la section). Une importance rendue possible justement grâce à l'utilisation des machines et à la mise en place d'une organisation scientifique du travail, amenant une décomposition et une simplification des tâches du travail. Les Forges et la Précision comprennent respectivement 4,9 et 4,8 \% de cheminotes. Dans les Forges, encore une fois c'est la présence d'une machine, le pilon, qui permet l'implantation du travail féminin (la majorité des femmes sont "pilonnières »!). À la Précision, comme dans d'autres industries d'ailleurs, l'habilité des femmes est recherchée pour certaines fabrications délicates.

À l'opposé, la section des Ressorts n'emploie pas d'agent féminin. Au montage, elles ne sont que quatre aides $(1,3 \%)$, laissant leur place aux ajusteurs, ouvriers très qualifiés. La dureté du travail dans les Chaudronneries, aussi bien de cuivre que fer, les évince complètement (respectivement $0,9 \%$ et $1,8 \%$ de femmes). Mais globalement, les cheminotes restent largement minoritaires voir marginales dans les ateliers, ne représentant en février 1920 que 2,6 \% de l'effectif total des employés.

Finalement, les cheminotes ne sont pas dans les ateliers pour effectuer un travail d'ouvrier qualifié, mais bien celui d'un exécutant. La compagnie précise qu'« un emploi intensifié du personnel féminin et l'appoint des prisonniers de guerre n'ont pu suffire à compenser [le] déficit [de personnel], surtout en ce qui concerne les ouvriers d'art affectés à l'entretien des machines et du matériel roulant $»^{20}$ et que « vers la fin 1916, sur les instances des Compagnies, le Gouvernement avait mis en sursis d'appel, pour renforcer les effectifs appauvris des ateliers et des dépôts, quelques ouvriers spécialistes appartenant à la réserve de

18- Union des syndicats du réseau PLM, 1918, op. cit.

19- Félix Mangini, «De l'emploi des femmes à l'ancienne compagnie des Dombes », RGCF, mai 1885 , p. 322.

20- C.A. P.L.M., 1917, p. 40-41. 


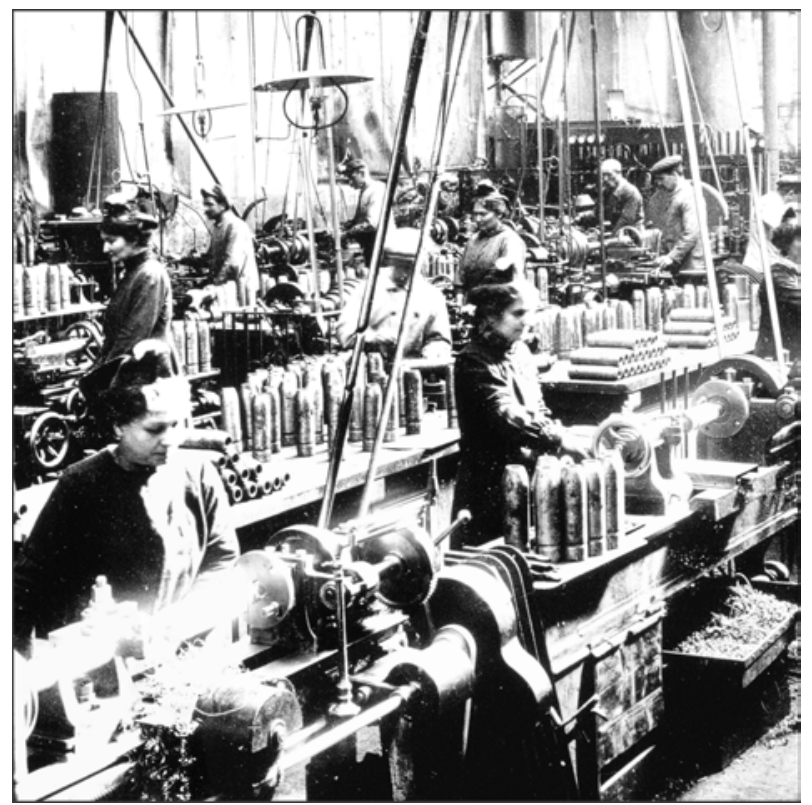

Figures 1 et 2. Femmes aux ateliers d'Arles. Coll. Museon Arlaten, musée départemental d'ethnographie, Arles, cl. J.-L. Mabit.

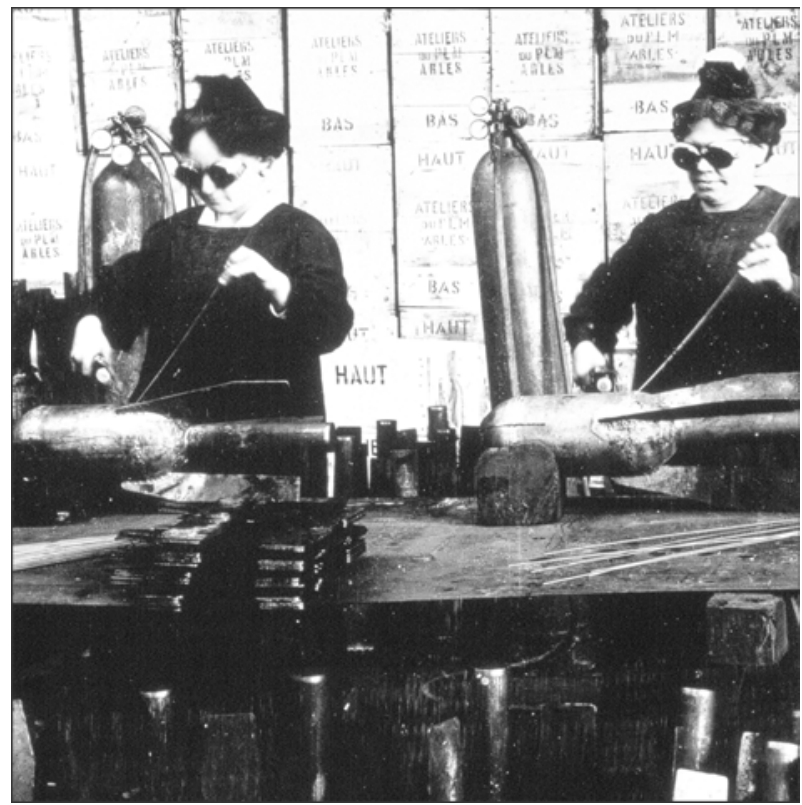


l'armée territoriale $»^{21}$. Si les femmes ne remplacent pas les ouvriers d'art, en revanche elles compensent le départ des agents les moins qualifiés et dans les mêmes conditions de travail.

\section{Les conditions de travail}

Se heurtant à un monde d'hommes, les cheminotes qui travaillent dans les ateliers doivent s'adapter. Rien n'est spécifique à la gent féminine, et, globalement, leurs conditions de travail ne diffèrent pas de celles de leurs collègues masculins. Depuis 1915, la durée d'une journée de travail est allongée pour tout le personnel de 10 à 11 heures. Pire, la compagnie, pour poursuivre l'effort de guerre et honorer ses engagements, décide de faire travailler ses ouvriers le dimanche. Ainsi, les cheminotes enchainent des semaines de travail de 77 heures, sans jour de repos, et cela sans compter celles qui acceptent de faire des heures supplémentaires. Face à ces pratiques, en 1918, des cheminots « déclarent que le travail fourni depuis trois ans par la journée de 11 heures constitue un effort suffisant, demander davantage serait un danger pour la santé et pourrait avoir des répercussions de maladie sur les privations d'alimentation supportées en ce moment $»^{22}$. D'autres demandent la « suppression du travail du dimanche, qui impose avec les heures supplémentaires, un surmenage inutile $»^{23}$. Si le dimanche n'est toujours pas chômé, en revanche le retour aux dix heures de travail est effectif à partir du mois d'août 1918, suite à un accord survenu entre le ministère des Travaux publics et la Fédération nationale des cheminots. Mais les cheminotes ne sont pas mieux loties que les autres femmes travaillant dans l'industrie car "une enquête de 1917 révèle qu'une femme sur quatre travaille plus de onze ou douze heures par jour, les autres un minimum de dix heures $»^{24}$.

En plus de longues journées, certaines femmes effectuent des tâches physiquement difficiles et pénibles. Ainsi en 1918, le secrétaire général du syndicat d'Arles interpelle le sous-ingénieur, chef des ateliers des machines d'Arles au sujet de « l'emploi des femmes dans les travaux

21- C.A. P.L.M., 1920, p. 28.

22- Syndicat d'Arles, copie de l'ordre du jour de la réunion du 17 avril 1918 du syndicat de Clermont-Ferrand.

23- Union des syndicats du PLM, Compte rendu du premier congrès de l'Union tenu à Avignon, le 5 et 6 mai 1917, 1917, Dijon, Imprimerie coopérative ouvrière, p. 36.

24- Louis-Henri Parias, Histoire générale du travail, tome 4 : la civilisation industrielle (de 1914 à nos jours), Paris, Nouvelle Libraire de France, 1961, p. 117. 
de force $»^{25}$. Des constatations répétées puisqu'elles entraînent les cheminots à demander «dans différents services, la suppression de la femme dans les travaux de force et dans les travaux de sécurité $»^{26}$. Mais, même en dehors des travaux de force, les conditions de travail, en général, sont dures : travailler dans le froid ou la chaleur des ateliers, dans la poussière créée par les machines, et aggravées par le non-respect de l'«Application intégrale des lois et décrets de protection du travail féminin et de l'hygiène dans les ateliers $»^{27}$.

Mais d'autres inquiétudes naissent chez les collègues masculins des travailleuses. En 1918, un délégué syndiqué dit : «Je serais partisan que l'on demande aussi d'éviter autant que possible la promiscuité des deux sexes pendant le travail. [...] Si parmi nous la plupart des gens sont assez corrects, il y en a quelques-uns qui ne le sont pas et il peut se produire des incidents ${ }^{28} \ldots$ » Un autre « propose que pour éviter les dangers de la promiscuité, les services féminins soient dirigés par des dames chefs de service $»^{29}$. Malaise d'un monde masculin pas encore habitué à côtoyer quotidiennement des femmes, difficulté partagée par une cheminote qui raconte : «que tous ces messieurs ont été avec moi d'une correction parfaite. Tous ont été gentils et serviables. Je me souviens cependant des premiers jours. Entre tous ces hommes, j'étais complètement perdue ${ }^{30}$. » Une incompréhension mutuelle qui rend les relations de camaraderie difficiles, d'autant qu'une autre barrière se dresse entre les hommes et les femmes : celle du salaire.

\section{À travail égal, salaire égal!}

Les femmes, comme tous les cheminots des ateliers, sont payées à la tâche. Malgré de multiples actions des syndicats, dès 1917, pour demander « La suppression du travail à la tâche [et la] mise au mois de tout le personnel avec [1] établissement d'une échelle de traitement $»^{31}$,

25- Lettre du secrétaire du syndicat d'Arles du 13 février 1918 adressée au sousingénieur, chef des ateliers des machines d'Arles. Le syndicat des cheminots d'Arles a conservé ses archives pour la période 1917-1920. Ces dernières sont consultables auprès de l'Institut d'histoire sociale régional des cheminots CGT-PACA.

26- Union des syndicats du réseau PLM, 1918, op. cit., p. 118.

27- Union des syndicats du PLM, 1917, op. cit., p. 36.

28- Union des syndicats du réseau PLM, 1918, op. cit., p. 120.

29- Ibid., p. 122.

30- Bruno Carrière, "Cheminote en 1917 », La Vie du Rail, n 1899 (23 juin 1983), p. 42. Cette personne a effectué toute sa carrière à l'Exploitation.

31- Union des syndicats du PLM, 1917, op. cit., p. 35. 
ce système perdure. Les cheminotes, «aides 》 ou « ouvrières ", touchent toutes un salaire identique de 0,61 franc de l'heure en 1918. Une seule exception dans cette uniformité de traitement des agents féminins, les « manœuvres » qui sont moins payées, avec un taux horaire de 0,58 franc, soit un salaire inférieur de 4,9\%. Une différence de traitement justifiée, tant par la direction que par les employés, en reconnaissance d'une différence du travail. Par ailleurs, à la création de l'échelle des traitements dans le statut de 1920, les manœuvres sont classés au premier échelon (dénommé A), alors que les conducteurs de machines-outils, comme les aides, appartiennent au troisième (appelé $\mathrm{C}$ ).

Les agents masculins prônent « l'unification des salaires pour toutes les femmes du fait qu'elles n'ont fait aucun apprentissage avant leur rentrée à la compagnie $»^{32}$. Du côté de la compagnie, la perception d'une somme identique journalière laisse présager qu'elle considère que toutes les femmes (hormis les manœuvres) remplissent les mêmes fonctions dans le processus de production et reconnait implicitement l'équivalence des métiers, au moins au niveau de la qualification et de l'aptitude requise.

Le taux horaire du travail féminin était de 0,55 franc de l'heure de 1915 à 1917, mais il est augmenté à 0,61 franc en 1918. À partir de ce moment, les travailleuses perçoivent donc un salaire supérieur de 9,8\% par rapport aux années précédentes. Par ailleurs, en 1917, des mouvements de grève ont lieu dans l'industrie d'armement pour réclamer une hausse des salaires afin, notamment, de faire face à l'augmentation du coût de la vie. Les cheminotes employées dans les ateliers de la Compagnie du PLM ont probablement bénéficié, elles aussi, des mesures qui en découlent. Nous ne pouvons pour autant affirmer qu'elles aient participé à ce mouvement. À cette rémunération s'ajoute une prime de cherté de la vie de deux francs par jour, à partir de juillet 1918. Une autre prime annuelle est octroyée en 1919 : celle de résidence de 300 francs qui est doublée, en 1920.

Au cours d'une journée, les femmes «aides » ou «conductrices de machines-outils » gagnent 6,05 francs pour 11 heures de travail en 1917 ; leur salaire passe à 6,10 francs en 1918 , mais pour dix heures de travail. L'augmentation du taux horaire a compensé la perte de l'heure de travail. En comparaison des salaires distribués dans les autres industries de guerre notamment, les cheminotes semblent plutôt mieux payées. Durant le conflit, les ouvrières de l'armement de Saint-Étienne touchent

32- Syndicat d'Arles, lettre adressée au secrétaire général Dussaix, datée du 30 avril 1918. 
entre «2,50 et 4 francs par jour $»^{33}$ pour dix heures de travail. En 1923, dans une usine de lampes, « des ouvrières sont payées par journée 0,60 à $0,75 \mathrm{~F}$ l'heure $»^{34}$. La même année, dans une glacerie, elles touchent également $« 0,75$ franc $»^{35}$. Les travailleuses des ateliers paraissent donc plus avantagées que leurs collègues « munitionnettes », avec des salaires supérieurs.

À l'intérieur des ateliers, leur condition est différente. Nous ne possédons aucun élément concernant le salaire des hommes entre 1914 et 1918. En revanche, nous savons qu'un « aide-ajusteur » embauché en janvier 1919 gagne 0,80 franc de l'heure. Si l'on compare l'écart de rémunération entre les femmes et les hommes pour cette période de 1918-1919, le taux horaire de salaire féminin est inférieur de $25 \%$ à celui des cheminots. Il est justifié indirectement par les propos répétés d'un délégué : « Notre ingénieur nous disait qu'il donnait $25 \%$ de moins au femmes, comme pourcentage de leurs salaires en raison, de ce que, dans l'industrie privée, ce $25 \%$ existait toujours ${ }^{36}$. » Une différence d'un quart qui est loin d'être négligeable en cette période d'inflation. Cet abattement est donc une pratique admise par tous les industriels, y compris par les chemins de fer, mais pour la même période, s'il «était de $18 \%$ en 1916 , pour les manœuvres des métaux de la région parisienne, [il] est à $31 \%$ en 1921. Il passe de 22 à $40 \%$ à Toulouse, de 16 à $37 \%$ au Havre $»^{37}$. Cette inégalité des salaires existe dans tous les services, « reconnue comme un état de fait : complément salarial pour [certaines cheminotes], gage d'indépendance financière pour les autres... [malgré une] égalité devant la tâche à accomplir $»^{38}$. Si cette différence de traitement mécontente les travailleuses, qui pour les mêmes conditions de travail perçoivent un salaire inférieur, elle dérange aussi les hommes qui y voient une concurrence déloyale à leur travail.

Le syndicaliste Chastagnier dit : « Eh bien camarades, il faut exiger que le travail égal soit à salaire égal pour les femmes. Oh ! nous savons bien qu'une femme ne produit pas, ne produira pas, ne peut pas produire

33- Évelyne Sullerot, Histoire et sociologie du travail féminin, Paris, Éditions Gonthier, coll. Grand Format Femmes, 1968, p. 141.

34- Annie Fourcaut, Femmes à l'usine en France dans l'entre-deux-guerres, Paris, Maspéro/ CRHMSS, 1982, p. 107.

35- Ibid., p. 113.

36- Union des syndicats du réseau PLM, 1918, op. cit., p. 143.

37- Edmonde Charles-Roux (sous la direction de), Les Femmes et le travail du Moyen-Age à nos jours, Paris, Éditions Temps actuels, 1975, p. 176.

38- Bruno Carrière, art. cit., p. 44. 
autant qu'un homme. Mais néanmoins, puisque la Compagnie veut les employer exactement comme nous, pour éviter la confusion sur le marché du travail, il faut que la Compagnie les paie comme nous, car camarades, si nous n'arrivons pas à imposer aux Compagnies le salaire égal, la Compagnie embauchera des femmes autant qu'elle le pourra et nous resterons peut-être, comme dit l'autre, en carafe. Mais nous aurons des difficultés pour faire triompher nos revendications futures si le marché de la femme est mis en présence du nôtre ${ }^{39}$. » Un autre d'ajouter : "dans les dépôts, [les travailleuses] sont appelées à remplacer les nettoyeurs. Eh bien, j'estime que si la femme ne nettoie pas la machine aussi vite peut-être que le nettoyeur lui-même, on doit la payer la même chose parce que notre prix de travail ne doit pas craindre la concurrence dans cette catégorie de notre industrie. Il est de même au Matériel. ${ }^{40}{ }$ »

\section{Les femmes, des militantes}

Face aux conditions de travail difficiles et à cette différence de rémunération, les syndicats décident de s'intéresser aux travailleuses. Un délégué syndical précise : « nous devons soutenir les femmes et en même temps essayer de les attirer à nous, au moins celles qui travaillent avec nous, car nous ne devons pas les laisser isolées face à la Compagnie ${ }^{41}$. » Et le président de séance d'ajouter « une propagande intensive devra être faite dans tous les milieux cheminots en faveur de l'obtention par les femmes de tous leurs droits ${ }^{42}$. Apparemment le syndicat CGT des chemins de fer d'Arles n'a pas attendu le congrès pour mobiliser les cheminotes, car quelques-unes payent déjà une cotisation en janvier 1918 et la totalité des femmes de notre échantillon en sont membres en 1919$1920^{43}$.

Dès son premier congrès de 1917 , le discours de l'Union des syndicats PLM est très offensif. Il pose comme revendications pour le service du Matériel l'« Application intégrale des lois et décrets de protection du travail féminin et de l'hygiène dans les ateliers [et l']application du principe spécialement en ce qui concerne la femme : à travail égal, salaire égal »4. Des thèmes repris jusqu'en 1919: «Application sans

39- Union des syndicats du réseau PLM, 1918, op. cit., p. 119.

40- Ibid., p. 119-120.

41- Ibid., p. 120.

42- Ibid., p. 121.

43- Elles sont clairement identifiables par une séparation au sein du registre, conséquence du paiement d'une cotisation mensuelle à moitié prix, d'un montant de 0,25 franc.

44- Union des syndicats du PLM, 1917, op. cit., p. 36. 
restriction, des lois et décrets de protection du travail féminin et de l'hygiène, adoption par la Compagnie, du principe, en ce qui concerne la main-d'œuvre féminine, "à travail égal, salaire égal"45. » Preuve que les volontés des syndiqués, hommes ou femmes, tardent à être satisfaites par la compagnie.

Mais un problème plus inquiétant se fait jour avec l'approche de la fin du conflit : celui de l'avenir des femmes au sein de la compagnie. D’après un cheminot, « Il faut regarder après la guerre, et après la guerre, il sera difficile de placer les ouvrières. Il faut essayer de les organiser pour imposer leur existence aux capitalistes $»^{46}$. En effet, dès la fin du conflit, la compagnie doit faire face à l'arrêt de la production de matériel militaire et à la reconversion de ses activités. La réparation et la maintenance du matériel ferroviaire requièrent plus de compétences, et donc plus de personnel qualifié. S'ajoute la démobilisation des combattants qui ramène vers les ateliers les agents désireux de (re)trouver un emploi ; ils concurrencent, désormais, le travail des femmes.

\section{Avec la fin de la guerre, une main-d'œuvre gênante}

En 1919, le PLM dresse le bilan suivant : « Les sources habituelles de recrutement étant taries, nous avons dû employer, à titre provisoire, en même temps que d'anciens Agents, des femmes en trop grand nombre, des prisonniers de guerre, des étrangers de toute nationalité, et la valeur technique et morale de notre personnel s'en est affaiblie malgré nos efforts pour la remonter ${ }^{47}$. » Transparait l'affirmation que les femmes sont une "main-d'œuvre provisoire», et surtout qu'elles sont désormais en " trop grand nombre ». Des propos qui ne laissent supposer qu'une seule issue : la compagnie veut se séparer d'au moins une partie, si ce n'est de l'intégralité, de son personnel féminin.

Les registres du syndicat d'Arles indiquent qu'entre août 1918 et décembre 1919105 travailleuses quittent les ateliers. Alors que 41 cheminotes sont indiquées " démissionnaires », 32 « quittent la compagnie », 19 « sortent» et trois sont "décédées ». De son côté, la compagnie « remercie » sept ouvrières et en "congédie » trois autres, donc $90,5 \%$ de départs seraient volontaires. Ainsi, avec le retour des

45- Union des syndicats du PLM, Compte rendu analytique du Congrès de Marseille, des 27, 28, 29, et 30 mars 1919, s.d., Villeneuve-Saint-Georges, Imprimerie de l'Union Typographique, p. 227.

46- Union des syndicats du réseau PLM, 1918, op. cit., p. 120.

47- C.A. P.L.M., 1920, p. 28. 
hommes, beaucoup de femmes regagnent leur foyer de leur plein gré ou non. Mais, apparemment, le PLM peut difficilement se séparer de celles qui ne le désirent pas.

L'obtention de leur commissionnement les protège et rend leur licenciement plus difficile. Alors le réseau tente de négocier leur départ avec le syndicat : « On [n']a pas envisagé le licenciement total, mais pour

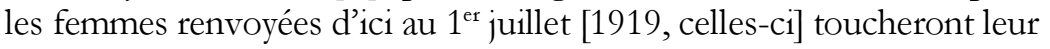
prix de journée $+3 \mathrm{~F}$ [multiplié] par le nombre de mois de présence à la Cie. Pas d'indemnités pour celles sortant volontairement et celles renvoyées après le $1 / 7^{48}$. » Le secrétaire général du syndicat ajoute qu'« il $\mathrm{y}$ a des chances que les femmes ne [soient] renvoyées qu'après le $1^{\text {er }}$ juillet pour esquiver l'indemnité ce qui constituerait un vol, mais que nous ferons en sorte, [que] cette indemnité soit donnée pour tous les licenciements de l'année courante ${ }^{49}$. Nous n'avons aucune idée de la portée de cette mesure, mais après janvier 1920, quelques femmes, dont nos 39 cheminotes, travaillent toujours aux ateliers.

La compagnie n'attendra pas longtemps pour avoir un bon prétexte pour se séparer de ce personnel féminin, apparemment encombrant depuis la fin du conflit. Le déclenchement de la grève de mai 1920 et la probable participation des travailleuses au mouvement vont amener leur révocation. Par une stratégie de fermeture de ses ateliers, le PLM licencie tous les cheminots. À partir du premier juin, il réembauche les ouvriers par convocation individuelle, mais les femmes ne font pas partie de la liste des « rappelés ». Toutes les cheminotes de notre échantillon portent sur leur dossier la mention « révoquée le 24 mai 1920 ». Dans certaines sections syndicales, où la liste du personnel est particulièrement bien tenue, en face de chaque nom de travailleuse rayé d'un trait rouge figure la mention " révoquée ». On peut alors penser qu'il s'agit d'une révocation systématique des travailleuses des ateliers. Une stratégie qui a permis au PLM de mettre fin à l'emploi des femmes dans ses ateliers, ainsi qu'à celui d'agents jugés gênants. Cette mesure provoque le retour dans leur foyer des dernières travailleuses qui ne l'avaient pas choisi.

Ces derniers départs sont certainement une satisfaction pour la compagnie, mais ne le sont-ils pas aussi pour certains agents masculins qui craignent « les femmes [qui] sont appelées à [les] concurrencer $»^{50}$ ? L'un d'eux dit que « nous estimons, nous, cheminots, que nous avons le

48- Syndicat d'Arles, réunion générale du 29 avril 1919, feuille 1.

49- Ibid., feuilles 1 et 2 .

50- Union des syndicats du réseau PLM, 1918, op. cit., p. 120. 
droit de gagner notre vie, que nous versons pour une retraite et qu'il ne faut pas que la femme vienne nous chasser $»^{51}$, et un autre de demander «qu'elles ne soient pas concurrentes des hommes sur le marché du travail ; et pour cela exig[e] qu'elles soient traitées comme eux à tous les points de vue (à travail égal, salaire égal) $\rangle^{52}$. Discours ambigu du syndicalisme qui, d'un côté, veut protéger les femmes qui travaillent, mais, d'un autre côté, les perçoit comme des concurrentes.

Finalement, ces cheminotes n'ont été pour la compagnie qu'une main-d'œuvre d'appoint, embauchée aux ateliers d'Arles pour faire face à une situation exceptionnelle : la Grande Guerre et ses besoins spécifiques. Avec l'armistice et le retour des hommes, le réseau PLM se trouve à la tête d'un personnel féminin dont il n'a plus besoin et qu'il ne souhaite pas garder. Sans qualification, ni apprentissage proposé par la compagnie au cours de ces années de guerre, les femmes sont restées un personnel atypique et marginal dans un monde d'hommes. Les mentalités avaient certainement encore besoin d'évoluer pour accepter cet autre travailleur appelé « femme ».

51- Ibid.

52- Ibid., p. 122. 